\title{
Pay for Performance-Panacea or Pandora's Box? Revisiting an Old Debate in the Current Economic Environment
}

Christine Martin Bevilacqua

Student

Parbudyal Singh

Associate Professor

York University

\section{New research in neuroeconomics suggests that money may be a more powerful motivator than previously thought, with important implications for incentive pay programs.}

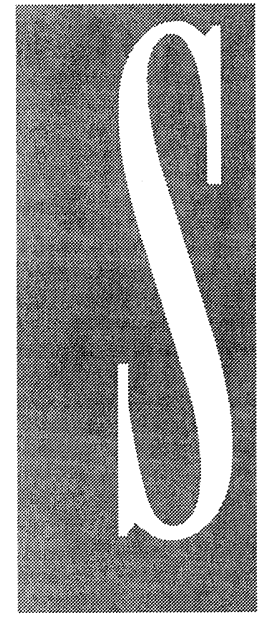

ince the fall of 2008, few individuals have been as vilified as senior employees in the disintegrating worldwide financial services sector. Executives at organizations such as Citigroup, or American International Group (AIG) in the United States, Bradford and Bingley in England, Fortis in Belgium or Glitner Bank in Iceland have all been forced to seek government assistance as their operations failed in the midst of an unprecedented international financial crisis.

However, even as these bailouts were taking place, incentives that had been previously negotiated were being paid out. This resulted in an enormous backlash from the media, scholars, politicians and citizens who failed to see how the individuals within these organizations should continue to reap the rewards of previously negotiated incentive plans in the face of dismal organizational performance. Although pay-for-performance systems have been seen as effective methods of linking individual performance and rewards, the current crisis has revealed that the problems associated with the design of such systems may not be as clear cut as previously thought.

Pay-for-performance systems are frementlv used by companies in an effort to link c sation to improved employee performance. In fact, a recent study by Hewitt suggested that $77 \%$ of all U.S. companies utilize at least one pay-forperformance plan within their compensation

Keywords: pay for performance; incentives; neuroeconomics; compensation design 
systems. ${ }^{1}$ Although now a well-established set of tools in the arsenal of compensation design professionals, these plans vary significantly depending on the context in which they are being deployed.

Consideration is usually given to the organization's environment, the competitiveness of the market in which it does business, environmental munificence (the degree to which resources are abundant or scarce), environmental turbulence and uncertainty, industry, size, age, product life cycle, culture, degree of unionization, nature of the work performed and region. ${ }^{2}$ Yet for all of the consideration given to the design, there are serious flaws that continue to exist in pay-for-performance systems.

The frequently unanticipated problem with many pay-for-performance systems is that they may be more effective than anticipated by their designers. In today's cash-focused culture, where new research suggests that money may have similar influences on individual actions as drugs or sex, the unexpected impact of plans that reward certain behaviors with cash is perhaps more than first thought. Because money has been shown to act as both an incentive when people understand that a particular action leads to obtaining money and as a reinforcer of the actions that led in the past to acquiring money, ${ }^{3}$ it is uniquely positioned to affect behavior in ways perhaps unanticipated by most practitioners.

In this article, we revisit the pay-for-performance debate, with a focus on individual incentives, especially as it has attracted so much attention in the current economic mess. The focus is on individual incentives because many of the criticisms do not apply to some group and organizational plans, such as gain sharing and profit sharing. In the next section, the article provides an overview of the traditional arguments for and against payfor-performance systems. It then discusses a new perspective on the use of money to motivate behavior. Finally, it discusses lessons and implications for managers.

\section{Arguments Supporting Individual Plans}

Proponents of individual incentive programs typically point to the tremendous growth in the use of such plans in compensation design as evidence of the positive impact incentive pay can have on organizational performance (see Exhibit 1 for some data on the trend). As a result of the

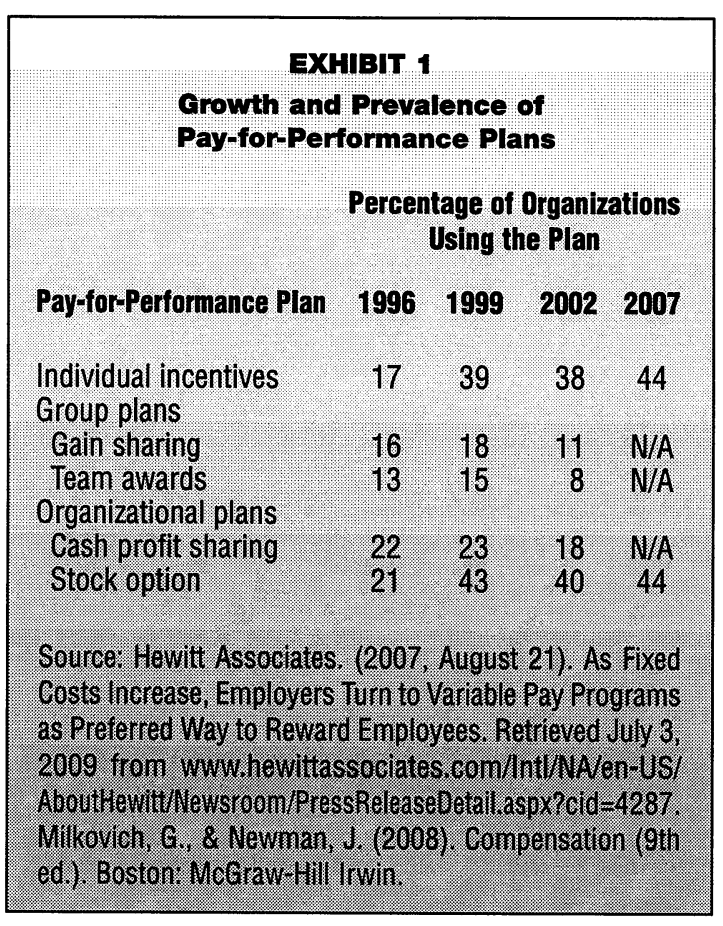

widely held belief that such plans offer significant opportunities for improved employee performance, incentives have become a larger portion of total executive pay and have become increasingly part of the compensation design in a wider range of employee levels and categories.

In turn, an entire field of compensation consulting practices have sprung up to aid organizations in developing elaborate incentive plans that strategically link to human resource management systems with the goal of improving the overall performance. In addition to simply pointing to the prevalence of such systems, supporters typically offer four key arguments in support of the use of incentive based pay.

The first, which is based on expectancy theory, suggests that employees will be motivated to work harder to achieve greater financial rewards. According to this argument, incentive plans typically link improved levels of employee outputs (e.g., sales, service, widget production) to an increase in pay. Incentives based on the achievement of goals that are achievable and implemented in a culture of trust drive behaviors required by the organization.

Second, they suggest that incentive plans can be effectively utilized to align the actions of individual employees with the goals of the organization. In most complex organizations, employees 
are called on to perform a wide variety of tasks. However, as a result of the increasing complexity of work, these employees may have a fair amount of latitude in the manner, order and timing of such tasks.

In addition to making decisions regarding the ordering of work, they may also be able to decide to apply or not to apply discretionary effort to subtly affect their output beyond the minimal expectations of the role. This argument suggests that by incenting certain behaviors, employees are more likely to differentiate their efforts to focus on certain activities over others in expectation of rewards.

Employers who track employee engagement or other similar satisfaction levels within their organizations often carefully monitor their staff's perception of the fairness of the organizations' policies and practices. Such organizations have often gone on to draw links between their positive employee engagement scores and decreased employee turnover, improved customer satisfaction and even increased financial performance. These employers believe that it is important for their employees to perceive that they are fair in their practices, including those related to compensation. Thus, from an equity perspective, pay-forperformance plans enhance employees' perceptions of fairness by providing greater rewards to those employees who have expended greater efforts.

Furthermore, reinforcement theory posits that employees will continue to exhibit certain positive behaviors when they are rewarded for doing so. Incentive plans that continue to reward certain behaviors consistently over time eventually hard wire certain activities or performance levels into the day-to-day actions of employees.

Finally, there is a large body of empirical research that suggests that individual incentives and other pay-for-performance plans contribute to increased employee productivity and organizational performance. ${ }^{4}$

\section{Arguments Against the Plans}

There is also a large body of literature on the problems associated with the use of pay-for-performance plans. Yet given the popularity of such systems, it is useful to remind practitioners about some of the potential perils.

First, to reward individual performance, managers must have an effective method to measure such performance, if it can be measured at all.
However, in most jobs, individual contributions are difficult to identify and/or performance is difficult to objectively measure. As a result, highly subjective ratings, especially those of supervisors, may be used instead of more measurable or objective ones.

Second, not only are pay-for-performance systems difficult to measure, but also they may actually decrease an employee's natural willingness to work. Rather than contributing to the success of their team or the organization itself because of the inherent motivation of the activities involved in the work itself, employees may become oriented to only working toward actions that are associated with rewards. In addition to the potential negative impact on intrinsic motivation, such activities may also decrease group cohesion as employees compete for limited resources or question the return of actions that seem purely social in nature.

Third, even when methods are in place to measure individual performance, managers are often extremely reluctant to rate their employees differently. They fear that the employees in their group may be less willing to work as a team, may volunteer for additional work less frequently or may simply interact less positively with the manager. Further complicating this issue is the fact that in difficult economic times compensation budgets may be decreased or even cut midperiod, resulting in unanticipated changes or eliminations of plans and an overall distrust by all of those involved in participation or administration of the systems.

Fourth, an important factor in the acceptance of a pay-for-performance system is the employees' perception of the fairness of the system. In general, employees will accept only plans that clearly communicate the rewards associated with the efforts and where the employees do not anticipate that the employer will reduce payouts should they exceed the plans' expectations. Employees need to trust management and believe that they can achieve the goals set for them. Managers in turn need to set fair yet challenging goals to substantiate the cost of pay-for-performance plans.

The effectiveness of any pay-for-performance system rests in its ability to adequately motivate an employee to behave in the manner in which the system was designed to reinforce. In the end, the underpinning of any pay-for-performance system is the establishment of an accepted method of performance evaluation-one that is fair and transparent. However, methods of 


\begin{tabular}{|c|c|}
\hline \multicolumn{2}{|c|}{$\begin{array}{l}\text { EXHIBIT } 2 \\
\text { gainst Pay-for-Performance Plans }\end{array}$} \\
\hline Arguments For & Arguments Against \\
\hline $\begin{array}{l}\text { There is value in these plans evident in their increasing } \\
\text { organizational use }\end{array}$ & $\begin{array}{l}\text { It is difficult and sometimes impossible to effectively } \\
\text { measure performance }\end{array}$ \\
\hline $\begin{array}{l}\text { When properly structured, they drive behaviors aligned } \\
\text { with organizational strategy }\end{array}$ & They can diminish intrinsic motivation \\
\hline $\begin{array}{l}\text { They allow employees to share in the wealth created by } \\
\text { their work }\end{array}$ & $\begin{array}{l}\text { Managers are sometimes reluctant to provide accu- } \\
\text { rate feedback }\end{array}$ \\
\hline $\begin{array}{l}\text { The research suggests that they enhance employee sat- } \\
\text { isfaction and productivity and are associated with } \\
\text { improved organizational performance }\end{array}$ & They may affect employees' perceptions of faimess \\
\hline & They may have unintended effects \\
\hline
\end{tabular}

evaluation are often fraught with significant problems.

Acceptance of feedback may be affected both by individual employee differences and by organizational or contextual variables. Some of the potential individual differences include the employees' self-esteem level, whether they exhibit an internal or external locus of control and their overall feedback orientation, whereas a key contextual or organizational factor is the perceived level of fairness in the process. ${ }^{5}$

Finally, another hindrance to the effectiveness of pay-for-performance systems is found in the nature of the behaviors that are chosen by organizations to be rewarded. Often, pay-for-performance systems become encumbered by complex, multilayered formulae that attempt to reward such a large number of behaviors that the employees are unable to keep their priorities straight.

Other poorly thought out systems result in designs that actually reward behaviors other than what the program was designed to incent. This can be especially true when an employee's efforts at optimizing one objective results in decreasing returns on other objectives. Furthermore, interventions that use money to bolster motivation also presume that performance is under the control of the people who get the incentives, when the actual work environment may require a high degree of cross-functional activity or where the performance of the employees or the organization itself is highly influenced by external competitive forces. ${ }^{6}$

The above issues have been discussed in the extant literature, and the debate has been ongoing (see Exhibit 2 for a summary of the arguments for and against). However, research in the area of neuroeconomics adds a new dimension to the discussion.

\section{Physiological Effects of Money}

This literature suggests that humans may actually be physiologically hardwired to react to money, adding considerable weight to existing concerns that such plans may actually work too well within an organization. Because of this, employees may be motivated to excessively focus on doing what they need to do to gain the reward at the expense of doing things to help the organization, ${ }^{7}$ or they may even be further tempted to manipulate systems by inflating measures or indulging in gaming practices. ${ }^{8}$

Neuroeconomics is a combination of neuroscience, economics and psychology that attempts to explain how both rational and irrational decision making occurs in the brain. ${ }^{9}$ As part of this research, neuroeconomists have used new scientific techniques to illustrate the real-world application of traditional theory with their current research focused on the impact of certain stimuli on the brain.

Through the use of magnetic resonance imaging, neuroscientists are able to map brain activity to show electrical activity when neurons fire and discharge such chemicals as dopamine. The most recent research in this field has attempted to map exactly how the brain will react to certain stimuli.

This research has shown that significant activity took place in the nucleus accumbens when participants were in the presence of money and immediate monetary incentives were shown to be associated with immediate versus delayed 
rewards. This rather unexpected result surprised researchers. Unlike traditional views of money that suggest it is only a tool to obtain goods and services, this new finding suggests that money may in fact be a motivator unto itself.

The nucleus accumbens is part of the bridge that connects the two sides of the brain; it is part of the limbic system that controls emotions such as hope, euphoria, addiction, fury, fear, lust, sexual arousal and aggression and is activated in situations that involve reward and punishment. Using magnetic resonance techniques, scientists have found that monetary gain stimulates the same reward circuitry as cocaine-in both cases dopamine is released into the nucleus accumbens.

In contrast, and perhaps equally as important in terms of pay-for-performance design, a threat of financial loss can result in employees demonstrating the classical fight-or-flight reactions such as an elevated heart rate, an increase in blood pressure or an elevated level of alertness, more typically associated with physical attacks and threatened harm. When anticipated gains are not realized, dopamine levels decrease, resulting in negative physical reactions to the loss of income.

Researchers have further demonstrated that automatic emotional responses such as fear and greed often trump more controlled or higher level brain responses, bringing into question the notion that employees react to pay-for-performance plans in a rational manner. Given that these reactions are hardwired into human physiology, it seems unlikely that individuals will be able to overcome these natural reactions to the stimulus of money by way of intellect alone.

Although most of the current theories rely on the notion that man makes rational decisions, this research suggests that humans struggle between reacting to the emotions that cause them to crave rewards and a wish to demonstrate rational thought and decision making when faced with competing alternatives.

\section{Implications for Managers}

Although other researchers have identified that strong links between pay and performance can exacerbate problems having to do with risk aversion, decrease in intrinsic motivation and so on, this new research adds to the concern that employees in pay-for-performance situations can be tempted to manipulate such systems by inflating measures, indulging in gaming practices or demonstrating unethical or even illegal behaviors as a reaction to certain financial incentive structures. $^{10}$

Agency theory has significantly affected compensation design models. It suggests that a principal, or an owner or employer, contracts with an agent or manager to perform certain behaviors that will result in outcomes to meet the principal's goals. In an attempt to persuade the agents to exert their best efforts, principals agree to incentive programs to ensure that the agents or managers (and employees) act in the organization's best interests. These models further suggest that the interests of the principal and the agent are further aligned through the process of monitoring to ensure that expected performance is achieved and to reduce the chance of the agent to act opportunistically. ${ }^{11}$

Agency theory suggests that employees are likely to take advantage of situations where there is inadequate monitoring of the work performed or where the incentive program design does not properly align the rewards to the expected outcomes. Without these checks and balances, it becomes more likely that employees will engage in illegal behaviors.

However, in the context of the neuroeconomic research discussed, it would appear that employers who choose to make use of pay-forperformance systems need to be even more vigilant against their unintended consequences than even this theory suggests. Organizations would also have to decide whether the actions required to effectively monitor employees who are motivated to engage in illegal behavior may significantly outweigh any benefits that pay-forperformance systems may otherwise bring to the organization.

That such serious design flaws exist in incentive plans should come as no surprise to scholars of white-collar crime. Blatant manipulation of circumstances to gain wealth or power seems hardly surprising given the Enron scandal or the recent Ponzi schemes of Bernard Madoff.

Legal and illegal activities to manipulate shortterm results have been used by those involved to ensure that they earn high, and sometimes outrageous, levels of compensation. Given what we now know about the physiological reaction displayed by humans at the thought of the acquisition of wealth, perhaps the more significant question for practitioners is why such things do not happen more often. 
For organizations that continue to implement pay-for-performance plans, serious consideration should be given to the design of such programs to ameliorate the issues associated with using money as a reward. Designers of these plans should anticipate that any pay scheme that has sufficient worth to have a positive impact on performance is likely to incent at least a portion of the organization's population to consider how they might outwit the program design. ${ }^{12}$

Consequently, plan design should involve those with sufficient knowledge of the processes and practices to anticipate how less than scrupulous employees might work the program in their favor. Unfortunately, those typically involved in such design - consultants or management-may lack sufficient hands-on knowledge to do so effectively and thus should solicit appropriate feedback during the design phase.

Plan designs should also focus on longer term results rather than items that can be manipulated in the short term to produce the stated objectives. In particular, pay-for-performance programs should avoid paying out for results that can be better attributed to external factors, such as an overall gain in the market, than the efforts of the plan participants.

In addition to the measurement of items with a longer term focus, consideration should be given to including claw backs or forfeiture of future rewards should payments be made in the short term that later are determined to be based only on manipulation of certain factors within the control of the participant at the expense of more important organizational goals. Furthermore, serious public sanctions should be established against those who have blatantly gamed the system.

Incentive plans should also be deigned to include a real downside risk if organizational results are not achieved. For example, plans that require that the participants hold a minimum level of equity, some through purchases, for a period of time-possibly including a period beyond their tenure-would help to ensure that employees in these firms focus beyond their own short-term interests.

Those in positions of governance of such plans should take a more active role in the monitoring to ensure that the goals of the program actually manifest themselves in the manner anticipated. Plan design should anticipate the time required for regular monitoring and adjustment by management, so that this important part of the process is not avoided by those who may prefer to shun evaluation of staff.
Governance committees will need to ensure that sufficient transparency and communication exist to counteract the natural temptation of investors, who may know relatively little about the business, to focus on single, easy-to-understand metrics such as quarterly profits and share prices, which offer more opportunity for internal manipulation. External and internal communication of plan design should focus on providing clear information and links among the goals of the compensation design and results. Rather than rubber-stamping extravagant compensation plans presented to them by management, boards of directors need to be held accountable for the success or failure of the compensation systems they endorse.

Because boards themselves can be accused of being systemically biased as a result of their inherent relationships and structure, some organizations may benefit from the solicitation of feedback on plan design from those who may be considered more neutral evaluators, such as institutional investors or those who support "sayon-pay" initiatives. In the end, boards themselves may need to be better constructed so that their members are more knowledgeable about the workings of the industry in which the company operates and can therefore offer more to the process of design than tacit approval of the recommendations of management or consultants.

Finally, organizations should not depend on incentives or pay-for-performance plans as a sole or even the most potent motivator of desired employee behavior. Ideally, these systems should complement other, and perhaps more important, facets of organizational life, including welldesigned jobs and workplaces, employee autonomy and involvement in their work, effective leadership and fair and just work systems. If an organization solely depends on incentives for employee motivation, it may be in more trouble than it realizes.

\section{Conclusions}

Pay may be far more significant a motivator than ever indicated by prior research on motivational theory. In fact, contrary to earlier beliefs, evidence suggests that pay is capable of fulfilling higher order needs as well as lower order needs such as food, security, social relations, esteem and even self-actualization needs. ${ }^{13}$ Researchers in the fields of sociology and social psychology have further expanded our understanding of the role of money as a marker of social status and an 
indicator of the possessor's general wealth, quantity of possessions and consumption. ${ }^{14}$

The recent economic downturn has illustrated the powerful potential impact of a loss of such status on even those who seem logically insulated by their wealth. An example of this was the suicide of German billionaire Adolf Merckle. His death was attributed to shame rather than its impact on his livelihood as he remained an extraordinarily wealthy person even after his companies suffered heavy losses. ${ }^{15}$

Evidence of the increasingly complicated role money plays in society is now being demonstrated by researchers in the neuroeconomics field. The findings that individual reactions to programs designed to motivate certain behaviors may be far less rational than previously considered and that reactions to monetary gains could be more similar to reactions to sex or drugs have critical implications for organizations that depend on pay-forperformance methods to incent the actions of their employees. The bottom line is that money may be an even more potent motivator than we thought; thus, compensation professionals must use incentives with much more care.

\section{Notes}

1. Pfeffer, J., \& Sutton, R. I. (2006). What's wrong with pay-for-performance. Industrial Management, 48(2), 12-17.

2. Minceli, M. P., \& Heneman, R. L. (2000). Contextual determinants of variable pay plan design: A proposed research framework. Human Resource Management Review, 10(3), 289-305.

3. Lea, S. E. G., \& Webley, P. (2006). Money as a tool, money as drug: The biological psychology of a strong incentive. Behavioral and Brain Sciences, 29, 161-209.

4. See Pfeffer \& Sutton (2006); Beer, M., \& Cannon, M. D. (2004). Promise and peril in implementing pay-for-performance. Human Resources Management, 43(1), 3-35.

5. McCarthy, A., \& Garavan, T. N. (2007). Understanding acceptance of multisource feedback for management development. Personnel Review, 36(6), 903-917.

6. Pfeffer \& Sutton (2006).

7. Beer \& Cannon (2004).

8. Rynes, S. L., Gerhart, B., \& Parks, L. (2005). Personnel psychology: Performance evaluation and pay for performance. Annual Review of Psychology, 56, 571-600.

9. Hannon, K. (2007). Review: Greed feels good, so watch out. Retrieved July 3, 2009 from www.usatoday.com/money/books/2007-0909-your-money-your-brain_n.htm. For details of this research, see Dubner, S. J. (2009). This is your brain on prosperity: Andrew Lo on fear, greed and crisis management. Retrieved July 2, 2009 from http://freakonomics.blogs. nytimes.com/2009/01/09/this-is-you-brainon-prosperity-andrew-lo-on-fear-greed-andcrisis-management/. Henschel, H. (2005). Beyond behavioural finance-The neuroscience of investment behaviour. Retrieved July 3, 2009 from http://www.cfa.ac.za/Neuroscience _SA.pdf. Lea \& Webley (2006); Lo, A. W., Dmitry, V. R., \& Steenbarger, B. N. (2005). Fear and greed in financial markets: A clinical study of day-traders. Cognitive Neuroscientific Foundations of Behavior, 95(2), 354-359; Milner, B. (2009). This is your brain. This is your brain on money. Retrieved March 10, 2009, from www.reportonbusiness.com.

10. Davis, J. L., Payne, G. T., \& McMahan, G. C. (2007). A few bad apples? Scandalous behaviour of mutual fund managers. Journal of Business Ethics, 76, 319-334; Rynes et al. (2005).

11. Ibid.

12. Hamblin, R. (2008). Regulation, measurement and incentives. The experience in the U.S. and U.K.: Does context matter? Journal of the Royal Society for the Promotion of Health, 128(6), 291-298.

13. Rynes et al. (2005).

14. Lea \& Webley (2006).

15. Kirschbaum, E. (2009, January 11). Shame not financial loss, prompts very rich to commit suicide. Gazette (Montreal), p. A7.

Christine Martin Bevilacqua is a student in the executive master's program in human resource management at York University, Canada. In addition to her academic experience, she has worked in a variety of senior human resource roles in the retail, financial services and hospitality industries.

Parbudyal Singh is an associate professor of human resource management at York University, Toronto. He has published more than 60 refereed articles, many in top journals such as Industrial Relations, Human Resource Management Review, Journal of Vocational Behavior and_Management International Review. His research focuses on compensation and other human resource management issues. 